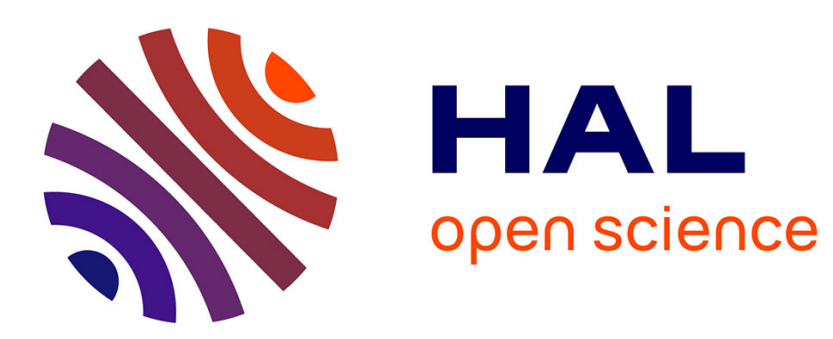

\title{
Optimisation d'un amortisseur d'une machine à courant continu : amélioration de la commutation
}

\author{
E. Peuro, M. Gabsi, M. Lécrivain, J. Rialland
}

\section{To cite this version:}

E. Peuro, M. Gabsi, M. Lécrivain, J. Rialland. Optimisation d'un amortisseur d'une machine à courant continu: amélioration de la commutation. Journal de Physique III, 1993, 3 (5), pp.961-972. 10.1051/jp3:1993172 . jpa-00248974

\section{HAL Id: jpa-00248974 https://hal.science/jpa-00248974}

Submitted on 1 Jan 1993

HAL is a multi-disciplinary open access archive for the deposit and dissemination of scientific research documents, whether they are published or not. The documents may come from teaching and research institutions in France or abroad, or from public or private research centers.
L'archive ouverte pluridisciplinaire HAL, est destinée au dépôt et à la diffusion de documents scientifiques de niveau recherche, publiés ou non, émanant des établissements d'enseignement et de recherche français ou étrangers, des laboratoires publics ou privés. 
Classification

Physics Abstracts

86.30D

\title{
Optimisation d'un amortisseur d'une machine à courant continu : amélioration de la commutation
}

\author{
E. Peuro, M. K. Gabsi, M. Lécrivain et J. F. Rialland \\ Laboratoire d'Electricité Industrielle, CNAM, 292 rue Saint Martin, 75141 Paris Cedex 03, \\ France
}

(Reçu le 26 juin 1992, accepté le 9 février 1993)

\begin{abstract}
Résumé. - L'introduction d'un circuit amortisseur dans une machine à courant continu, alimentée par des courants ondulés, améliore notablement son fonctionnement. On présente dans cette étude, une méthode d'optimisation de cet enroulement supplémentaire. La f.e.m. de transformation générée par l'enroulement inducteur dans la section en commutation, les pertes par effet Joule dans l'amortisseur et l'inducteur, ainsi que le temps de réponse dynamique de la machine sont les critères de l'optimisation. Leurs variations en fonction de la section totale de l'enroulement amortisseur peuvent nous conduire à un amortisseur optimal.
\end{abstract}

\begin{abstract}
The introduction of a damper winding into a DC machine, fed by undulated current, significantly improves its efficiency. We present, in this paper, a method for optimizing this supplementary winding. The criteria for optimization are the following : i) the e.m.f. transformation generated, by the field winding, in the commutating coil, ii) the copper losses in the damper and inductor, iii) the dynamic response time of the machine. The variations of these parameters, in fonction of the total section of the damper, will lead to an optimal functioning of this damper.
\end{abstract}

\section{Introduction.}

Une machine à excitation série associée à un convertisseur statique est caractérisée par une commutation difficile [1]. En effet, l'ondulation du courant inducteur génère une f.e.m. de transformation dans les sections de l'induit [2], donc un courant supplémentaire dans la section en court-circuit, dégradant ainsi la commutation [3].

Afin de limiter les étincelles aux niveaux des balais, la solution adoptée jusqu'à nos jours consiste en l'utilisation d'une inductance de lissage de forte valeur et de convertisseurs fonctionnant à des fréquences élevées.

L'introduction d'un enroulement amortisseur dans une machine à courant continu vise à mieux l'adapter à l'alimentation par des convertisseurs statiques [4]. La ressemblance entre cette machine et le moteur synchrone devient ainsi plus prononcée.

L'enroulement amortisseur posé aux niveaux des pôles inducteurs s'oppose aux variations de flux et atténue, ainsi, la f.e.m. de transformation. 
L'optimisation de cet enroulement est basée sur trois critères :

- la valeur de la f.e.m. de transformation induite dans une section de l'induit ;

- les pertes par effet Joule dissipées dans l'amortisseur et les pertes supplémentaires dans l'inducteur (la machine étant alimentée en tension);

- la réponse dynamique du moteur équipé d'un tel amortisseur.

\section{Préliminaires.}

L'étude porte sur une machine à excitation série d'une puissance nominale de $4 \mathrm{~kW}$. Le circuit magnétique est entièrement feuilleté. Afin d'effectuer des essais de validation, la machine est équipée d'un amortisseur constitué de 20 spires, le bobinage inducteur d'origine en contenant 60.

L'optimisation de l'amortisseur nécessite de connaître les paramètres de la machine et, plus précisément, les variations des valeurs des inductances en fonction des composantes alternative et continue du courant. Les mesures montrent l'influence notable de la composante continue du courant et, à un degré moindre, celle de l'amplitude de la composante alternative $[5,6]$. Par contre, dans un domaine compris entre 10 et $1000 \mathrm{~Hz}$, la fréquence n'a pas d'influence significative sur les valeurs des inductances [5]. A titre d'exemple, la figure 1 montre les variations de l'inductance propre de l'inducteur série avec le courant.

Les courbes relevées sont modélisées par des polynômes de degré 3. Un programme de simulation permet alors d'obtenir les valeurs des différentes inductances pour toutes les valeurs des composantes alternative et continue du courant.

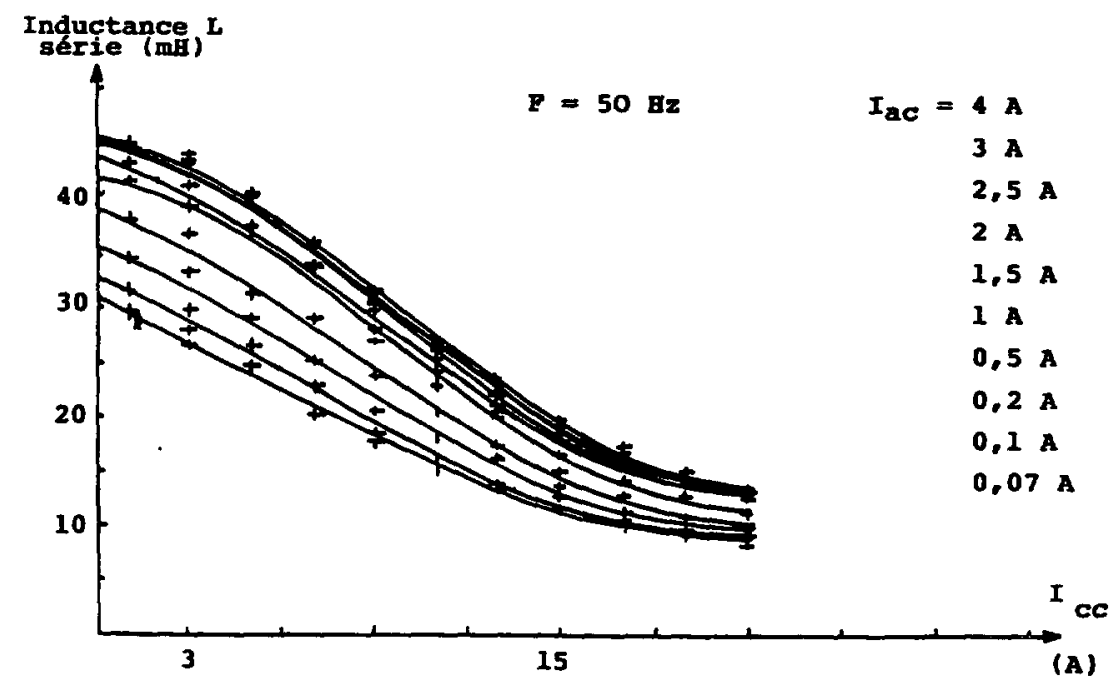

Fig. 1. - Variation de $L$ série en fonction de $I_{\mathrm{cc}}$ et de $I_{\mathrm{ac}}$.

[Measured curves of $L$ series against $I_{\mathrm{cc}}$ for a range of $I_{\mathrm{ac}}$ currents.]

\section{Analyse du fonctionnement.}

A 1'arrêt, la machine est assimilable à deux circuits (Fig. 2). Le premier, l'interrupteur K étant sur la position 1 ou 2, comprend une source de tension ( $u$ ou $E$ ) alimentant une inductance $L$ et une résistance $r$, rendant compte de l'induit et de l'inductance de lissage, en série avec 


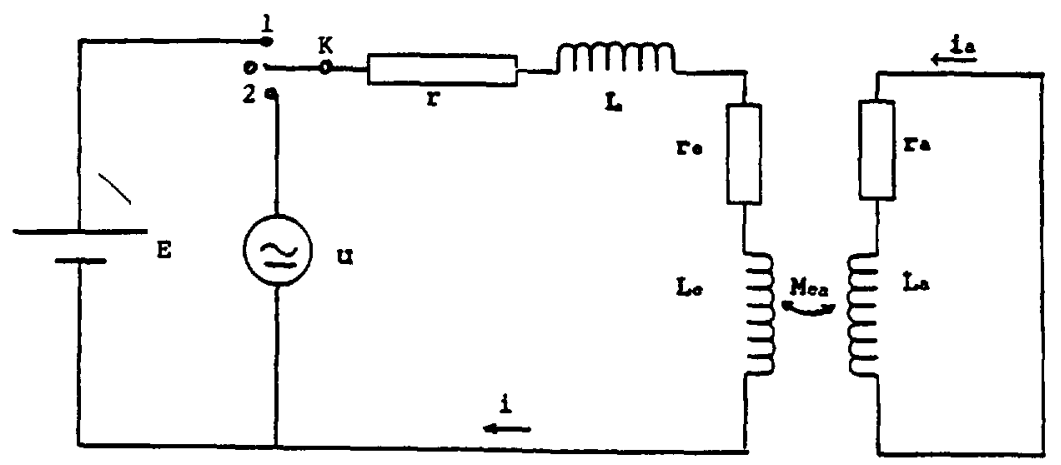

Fig. 2. - Schéma électrique équivalent de la machine à l'arrêt.

[Equivalent electrical circuit of the blocked machine.]

l'inducteur série représenté par sa résistance $r_{\mathrm{e}}$ et son inductance $L_{\mathrm{e}}$. En effet, comme l'induit est globalement découplé magnétiquement des enroulements série et amortisseur, il peut être assimilé à une simple inductance.

Le second circuit, associé à l'amortisseur $\left(r_{\mathrm{a}}, L_{\mathrm{a}}\right)$, est couplé au précédent par la mutuelle inductance $M_{\mathrm{ea}}$ entre l'inducteur série et l'amortisseur.

Le signal délivré par le générateur de tension est de la forme :

$$
u(t)=U_{\mathrm{cc}}+u_{\mathrm{e}}(t)
$$

avec

$$
u_{\mathrm{e}}(t)=U_{e} \cdot \sqrt{2} \cos \omega t,
$$

$U_{\mathrm{cc}} \quad$ valeur de la tension continue,

$U_{\mathrm{e}} \quad$ valeur efficace de la tension alternative,

$\omega$ pulsation de la tension alternative.

Ce générateur de tension représente pour notre calcul, une modélisation au sens du premier harmonique du convertisseur statique alimentant la machine.

Le courant $i$ dans le circuit inducteur est de la forme :

$$
i(t)=I+i_{\mathrm{e}}(t) .
$$

Les équations du système représenté par la figure 2 s'écrivent :

équation en continu :

$$
I=\frac{U_{\mathrm{cc}}}{r+r_{\mathrm{e}}}
$$

en alternatif (en utilisant la notation complexe) :

$$
\left\{\begin{array}{l}
U_{\mathrm{e}}=\left(r+r_{\mathrm{e}}\right) \cdot I_{\mathrm{e}}+j\left(L+L_{\mathrm{e}}\right) \cdot \omega \cdot I_{\mathrm{e}}+j M_{\mathrm{ea}} \cdot \omega \cdot I_{\mathrm{a}} \\
0=r_{\mathrm{a}} \cdot I_{\mathrm{a}}+j L_{\mathrm{a}} \cdot \omega \cdot I_{\mathrm{a}}+j M_{\mathrm{ea}} \cdot \omega \cdot I_{\mathrm{e}}
\end{array}\right.
$$


Il s'ensuit :

$$
\begin{aligned}
& I_{\mathrm{e}}=\frac{\sqrt{r_{\mathrm{a}}^{2}+L_{\mathrm{a}}^{2} \cdot \omega^{2}} \cdot U_{\mathrm{e}}}{\sqrt{\left[r_{\mathrm{a}} \cdot\left(r+r_{\mathrm{e}}\right)+\omega^{2} \cdot\left(M_{\mathrm{ea}}^{2}-L_{\mathrm{a}} \cdot\left(L+L_{\mathrm{e}}\right)\right)\right]^{2}+\omega^{2} \cdot\left[r_{\mathrm{a}} \cdot\left(L+L_{\mathrm{e}}\right)+L_{\mathrm{a}} \cdot\left(r+r_{\mathrm{e}}\right)\right]^{2}}} \\
& I_{\mathrm{a}}=\frac{M_{\mathrm{ea}} \cdot \omega \cdot U_{\mathrm{e}}}{\sqrt{\left[r_{\mathrm{a}} \cdot\left(r+r_{\mathrm{e}}\right)+\omega^{2} \cdot\left(M_{\mathrm{ea}}^{2}-L_{\mathrm{a}} \cdot\left(L+L_{\mathrm{e}}\right)\right)\right]^{2}+\omega^{2} \cdot\left[r_{\mathrm{a}} \cdot\left(L+L_{\mathrm{e}}\right)+L_{\mathrm{a}} \cdot\left(r+r_{\mathrm{e}}\right)\right]^{2}}}
\end{aligned} .
$$

Dans le cas de l'alimentation par un générateur de tension, la présence de l'amortisseur entraîne un accroissement des pertes par effet Joule dans le circuit inducteur.

Les pertes Joule dans l'amortisseur sont données par la relation :

$$
P_{\text {Jam }}=r_{\mathrm{a}} \cdot I_{\mathrm{a}}^{2}
$$

Les valeurs limites de ces pertes sont :

$$
\lim _{r_{\mathrm{a}} \rightarrow 0^{+}}\left(P_{\mathrm{Jam}}\right)=0 \text { et } \lim _{r_{\mathrm{a}} \rightarrow+\infty}\left(P_{\mathrm{Jam}}\right)=0
$$

avec un maximum $P_{\text {jam }}$ sax situé à :

$$
r_{\mathrm{a}}=\sqrt{\frac{\omega^{4} \tau^{2}\left(\frac{M_{\mathrm{ea}}^{2}}{L+L_{\mathrm{e}}}-L_{\mathrm{a}}\right)^{2}+L_{\mathrm{a}}^{2} \cdot \omega^{2}}{1+\omega^{2} \tau^{2}}} \quad \text { avec } \quad \tau=\frac{L+L_{\mathrm{e}}}{r+r_{\mathrm{e}}}
$$

Les pertes Joule du circuit inducteur dues à l'existence d'une composante alternative $\mathbf{i}_{\mathrm{e}}$ du courant s'écrivent :

$$
P_{\text {jexc }}=\left(r+r_{\mathrm{e}}\right) \cdot I_{\mathrm{e}}^{2} \text {. }
$$

Discussion sur les valeurs limites de $P_{\text {jexc: }}$

On considère, dans cette discussion, que le couplage magnétique entre $L_{\mathrm{e}}$ et $L_{\mathrm{a}}$ est parfait $\left(M_{\mathrm{ea}}^{2}=L_{\mathrm{e}} \cdot L_{\mathrm{a}}\right)$ et que, pour le cas de notre machine, $L_{\mathrm{e}}=9 . L_{\mathrm{a}}$. La relation (9) s'écrit alors :

$$
P_{\mathrm{jexc}}=\frac{\left(r+r_{\mathrm{e}}\right) \cdot\left(r_{a}^{2}+L_{a}^{2} \cdot \omega^{2}\right) \cdot U_{\mathrm{e}}^{2}}{\left[r_{\mathrm{a}} \cdot\left(r+r_{\mathrm{e}}\right)-\omega^{2} L_{\mathrm{a}} \cdot L\right]^{2}+\omega^{2}\left[L_{\mathrm{a}} \cdot\left(9 \cdot r_{\mathrm{a}}+r_{\mathrm{e}}+r\right)+L \cdot r_{\mathrm{a}}\right]^{2}} .
$$

Si $r_{\mathrm{a}} \rightarrow 0^{+}$, les pertes Joule du circuit d'excitation tendent vers :

$$
P_{\text {jexc }_{0^{+}}}=\frac{\left(r+r_{\mathrm{e}}\right) \cdot U_{\mathrm{e}}^{2}}{\left(r+r_{\mathrm{e}}\right)^{2}+L^{2} \omega^{2}}
$$

En considérant maintenant le cas où $L$ tend vers 0 (pas d'inductance de lissage et une très faible valeur de l'inductance de l'induit), on obtient:

$$
P_{\mathrm{jexc}_{0^{+}}}=\frac{U_{\mathrm{e}}^{2}}{r+r_{\mathrm{e}}}
$$

Ce qui revient à dire que, plus la résistance totale du circuit de l'inducteur est faible, plus les pertes par effet Joule sont élevées lorsque la résistance de l'amortisseur est faible. 
La présence de l'inductance de l'induit si elle est élevée, ou d'une inductance de lissage, atténue considérablement le phénomène. Si $r_{\mathrm{a}} \rightarrow+\infty$, les pertes Joule du circuit d'excitation tendent vers :

$$
P_{\text {jexc }_{+\infty}}=\frac{\left(r+r_{\mathrm{e}}\right) \cdot U_{\mathrm{e}}^{2}}{\left(r+r_{\mathrm{e}}\right)^{2}+\left(L+L_{\mathrm{e}}\right)^{2} \cdot \omega^{2}} .
$$

Ainsi, la variation des pertes Joule, dans le circuit d'excitation est décroissante en fonction de la résistance $r_{\mathrm{a}}$.

La f.e.m. de transformation dans une section de l'induit est caractérisée par la relation :

$$
E_{\mathrm{ts}}=j \omega \cdot\left(M_{\mathrm{es}} \cdot I_{\mathrm{e}}+M_{\mathrm{as}} \cdot I_{\mathrm{a}}\right) .
$$

La valeur efficace de $E_{\text {ts }}$ est :

$E_{\mathrm{ts}}=\frac{U_{\mathrm{e}} \cdot M_{\mathrm{es}} \cdot \omega \cdot \sqrt{r_{\mathrm{a}}^{2}+\left(L_{\mathrm{a}}-\frac{M_{\mathrm{ea}} \cdot M_{\mathrm{as}}}{M_{\mathrm{es}}}\right)^{2} \cdot \omega^{2}}}{\sqrt{\left[r_{\mathrm{a}} \cdot\left(r+r_{\mathrm{e}}\right)+\omega^{2} \cdot\left(M_{\mathrm{ea}}^{2}-L_{\mathrm{a}} \cdot\left(L+L_{\mathrm{e}}\right)\right)\right]^{2}+\omega^{2} \cdot\left[r_{\mathrm{a}} \cdot\left(L+L_{\mathrm{e}}\right)+L_{\mathrm{a}} \cdot\left(r+r_{\mathrm{e}}\right)\right]^{2}}}$.

En considérant les bobinages parfaitement couplés, les valeurs limites de $E_{t s}$ sont :

$$
\lim _{r_{\mathrm{a}} \rightarrow 0^{+}}\left(E_{\mathrm{ts}}\right)=0 \text { et } \lim _{r_{\mathrm{a}} \rightarrow+\infty}\left(E_{\mathrm{ts}}\right)=\frac{M_{\mathrm{es}} \cdot \omega \cdot U_{\mathrm{e}}}{\sqrt{\left(r+r_{\mathrm{e}}\right)^{2}+\left(L+L_{\mathrm{e}}\right)^{2} \cdot \omega^{2}}} .
$$

\section{Simulation du fonctionnement en présence de l'amortisseur : prédétermination-comparai-} son avec les mesures.

On considère que le générateur de tension délivre le même signal que précédemment.

On suppose que les courants alternatifs sont sinusoïdaux :

$$
\begin{gathered}
i_{\mathrm{e}}=I_{\mathrm{e}} \sqrt{2} \sin \Theta ; \quad i_{\mathrm{a}}=-I_{\mathrm{a}} \sqrt{2} \cos (\Theta-\varphi) \\
\Theta=\omega t \quad \text { et } \varphi=\operatorname{Arctg} \frac{L_{\mathrm{a}} \omega}{r_{\mathrm{a}}}
\end{gathered}
$$

avec

Les valeurs des inductances dépendent du flux engendré par la composante continue du courant et les courants alternatifs dans l'inducteur et l'amortisseur. La composante continue de courant est facilement calculable. Par contre, il est plus difficile de déterminer les courants alternatifs $i_{\mathrm{e}}$ et $i_{\mathrm{a}}$, ceux-ci dépendant des valeurs des inductances.

La somme vectorielle des courants $\mathbf{i}_{e}$ et $\mathbf{i}_{\mathrm{a}}$ correspond à un courant fictif $\mathbf{i}_{\mathrm{r}}$ représentant l'image du flux alternatif résultant dans la machine. Ainsi, les valeurs des inductances sont déterminées à partir de ce courant $\mathbf{i}_{\mathbf{r}}$

$$
i_{\mathrm{r}}=I+I_{\mathrm{r}} \sqrt{2} \sin (\Theta-\psi)
$$

avec $I_{\mathrm{r}}$ : valeur efficace du courant $i_{\mathrm{r}}, \psi$ : déphasage entre le courant $i_{\mathrm{e}}$ et le courant $i_{\mathrm{r}}$.

Pour $I$ connu, la valeur efficace $I_{\mathrm{r}}$ détermine les valeurs des inductances de la machine. En 
supposant que le couplage magnétique entre l'inducteur série et l'amortisseur est parfait, il s'ensuit : $n_{\mathrm{e}} i_{\mathrm{r}}=n_{\mathrm{e}} i_{\mathrm{e}}+n_{\mathrm{a}} i_{\mathrm{a}}$.

Moyennant un calcul simple [4], on montre que :

$$
I_{\mathrm{r}}=\sqrt{\left(I_{\mathrm{e}}-\frac{n_{\mathrm{a}}}{n_{\mathrm{e}}} \cdot I_{\mathrm{a}}\right)^{2}+2 \cdot \frac{n_{\mathrm{a}}}{n_{\mathrm{e}}} \cdot I_{\mathrm{e}} I_{\mathrm{a}}(1-\sin \varphi)} .
$$

La procédure de calcul est la suivante : partant de valeurs d'inductances correspondant à $I$, on calcule $I_{\mathrm{r}}$. On ajuste alors les valeurs des inductances et on redémarre le calcul jusqu'à obtenir la précision souhaitée:

Le programme de simulation du fonctionnement de l'amortisseur en générateur de tension permet d'observer l'influence de l'amortisseur en faisant varier l'un ou l'autre des paramètres $L_{\mathrm{a}}$ ou $r_{\mathrm{a}}$ (Fig. 3). Les pertes Joule du circuit d'excitation sont maximales à $r_{\mathrm{a}}=0 \Omega$ et diminuent rapidement avec l'augmentation de la résistance.

On peut constater que les pertes Joule maximales du circuit d'excitation sont relativement faibles. Cela est dô à la présence de l'inductance de l'induit qui limite considérablement ces pertes. Celles-ci s'élèvent pour $U_{\mathrm{e}}=30 \mathrm{~V}$, à plus de $200 \mathrm{~W}$ si $L_{\text {indurt }}=0$.

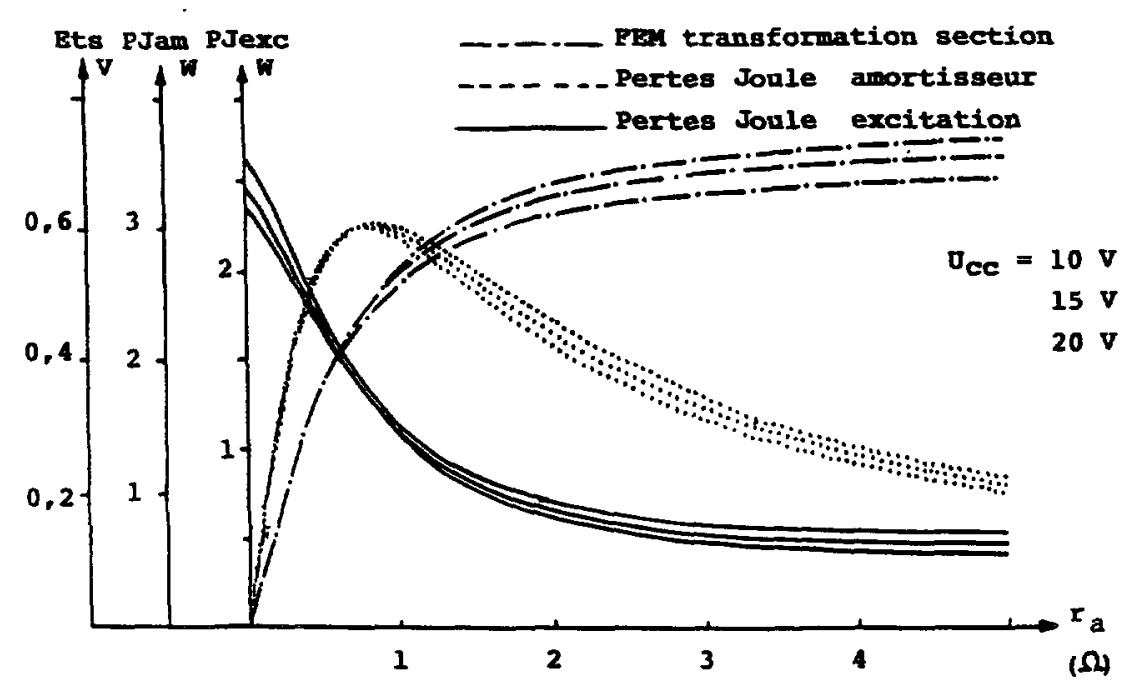

Fig. 3. - Influence de $r_{\mathrm{a}}$ sur la f.e.m. de transformation et sur les pertes Joule.

[The $r_{\mathrm{a}}$ influence on the transformer e.m.f. and on copper losses.]

\section{Validations.}

Les séries de mesures effectuées avec le circuit d'excitation alimenté par un générateur de tension ont permis de déterminer les pertes Joule de ce circuit et du circuit de l'amortisseur, ainsi que la f.e.m. de transformation dans une section, en fonction de la résistance $r_{\mathrm{a}}$ de l'amortisseur.

La figure 4 présente le schéma du circuit de mesure.

La section qui se trouve dans la zone de commutation est électriquement isolée du reste de l'enroulement de l'induit. 


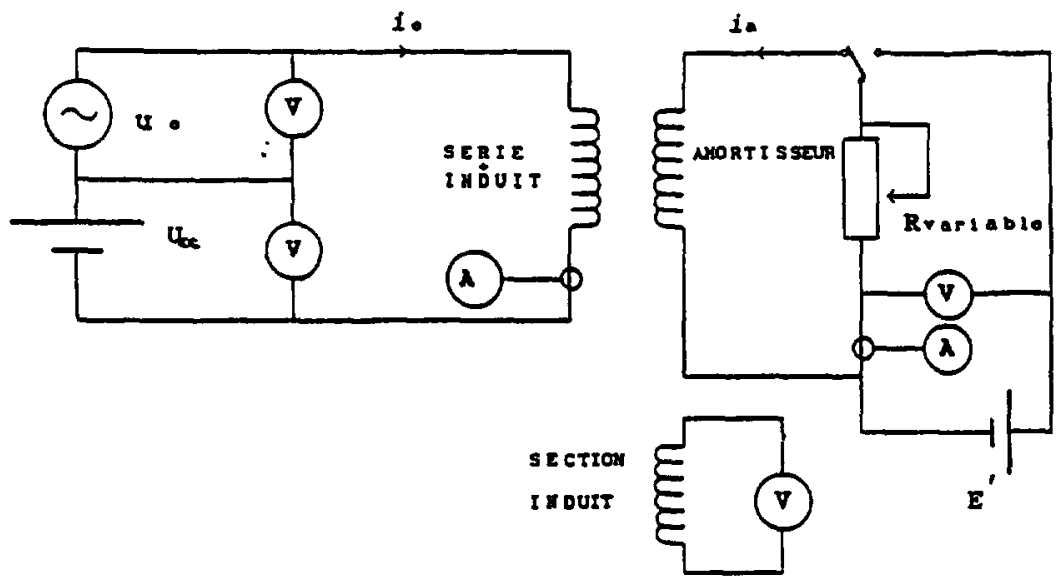

Fig. 4. - Schéma de mesure de la f.e.m. de transformation et de pertes Joule.

[Measurement circuit of the transformer e.m.f. and copper losses.]

La source de tension est réalisée à l'aide d'une source de tension continue placée en série avec un amplificateur de puissance commandé par un générateur de tension sinusoïdale.

On relève les valeurs efficaces $E_{\mathrm{ts}}, I_{\mathrm{e}}$ et $I_{\mathrm{a}}$ pour chaque valeur de $r_{\mathrm{a}}$.

L'ensemble des mesures effectuées ont été simulées en reprenant les mêmes valeurs des constantes $U_{\mathrm{cc}}, U_{\mathrm{e}}$, et en faisant varier $r_{\mathrm{a}}$ de 0 à $2 \Omega$.

Une comparaison entre les valeurs modélisées et les mesures, effectuée séparément pour les trois grandeurs étudiées $\left(P_{\text {jexc }} ; P_{\text {jam }} ; E_{\mathrm{ts}}\right)$, confirme bien la validité du modèle.

\section{Conclusion de l'étude en régime stationnaire.}

Les variations du rapport $\frac{L_{\mathrm{a}}}{r_{\mathrm{a}}}$ agissent non seulement sur les pertes Joule dans l'amortisseur et sur la f.e.m. de transformation, mais aussi sur les pertes Joule du circuit d'excitation.

L'analyse de ces pertes a démontré qu'elles restent faibles, même quand $r_{\mathrm{a}}$ tend vers 0 . Cela est, essentiellement, dû à la présence de l'inductance de l'induit.

Comme le modèle permet de faire varier la valeur de l'inductance de l'induit, il a été possible d'observer son influence sur les pertes. Ainsi, la variation de $L$ de $17 \mathrm{mH}$ (valeur moyenne de l'inductance de l'induit) à 0 produit une augmentation des pertes Joule du circuit d'excitation de $15 \mathrm{~W}$ à quelques $190 \mathrm{~W}$, pour un point de fonctionnement à $r_{\mathrm{a}}=0 \Omega$. Comme l'inductance de l'induit n'est pas nulle et que, dans le cas général, une inductance de lissage est associée à l'ensemble, le problème des pertes Joule du circuit d'excitation ne se pose pas.

La variation de la tension continue n'affecte pas particulièrement les pertes Joule dans 1'amortisseur ainsi que la f.e.m. de transformation.

Par contre, la valeur maximale de ces pertes est atteinte à une valeur de $r_{\mathrm{a}}$ plus petite. En effet, cette valeur n'est pas seulement liée à l'égalité $r_{\mathrm{a}}=L_{\mathrm{a}} \cdot \omega$, mais dépend aussi du rapport $\frac{L+L_{\mathrm{e}}}{r+r_{\mathrm{e}}}$ Plus ce rapport est élevé, plus le maximum des pertes Joule amortisseur est atteint rapidement.

Les performances de l'amortisseur sont fonction du rapport $\frac{L_{\mathrm{a}}}{r_{\mathrm{a}}}$; plus ce rapport est élevé, plus les performances sont améliorées. 


\section{Etude de l'amortisseur en régime dynamique.}

L'étude, en régime dynamique, du comportement de la machine en présence de l'amortisseur permet de mesurer l'impact de ce dernier sur le temps de réponse du moteur.

Un échelon de tension appliqué aux bornes du circuit d'excitation provoque une variation du flux dans le circuit magnétique. Le temps nécessaire pour que le flux atteigne sa nouvelle valeur dépend, en partie, de l'efficacité de l'amortisseur.

Un amortisseur parfait $\left(r_{\mathrm{a}}=0 \Omega\right)$ aurait pour conséquence d'empêcher toute variation de flux dans la machine.

L'étude qui suit pose les hypothèses suivantes :

- le couplage magnétique entre l'inducteur série et l'amortisseur est parfait, ainsi que celui existant entre l'inducteur série et une section de l'induit en position de commutation ;

- la mutuelle inductance entre l'induit et l'inducteur série est nulle ;

- la machine n'est pas saturée.

On se met dans le cas de petites perturbations, on considère donc les valeurs des différentes inductances constantes.

Considérons de nouveau le schéma de la figure 2 représentant le circuit électrique de la machine à l'arrêt et équipée de l'amortisseur.

A $t=0$, on ferme l'interrupteur $\mathrm{K}$ sur la position 1 . Les équations s'écrivent alors :

$$
\left\{\begin{array}{l}
E=\left(L_{\mathrm{e}}+L\right) \cdot \frac{\mathrm{d} i_{\mathrm{e}}}{\mathrm{d} t}+\left(r_{\mathrm{e}}+r\right) \cdot i_{\mathrm{e}}+M_{\mathrm{ea}} \frac{\mathrm{d} i_{a}}{\mathrm{~d} t} \\
0=M_{\mathrm{ea}} \frac{\mathrm{d} i_{\mathrm{e}}}{\mathrm{d} t}+L_{\mathrm{a}} \cdot \frac{\mathrm{d} i_{\mathrm{a}}}{\mathrm{d} t}+r_{\mathrm{a}} \cdot i_{\mathrm{a}} .
\end{array}\right.
$$

La résolution du système conduit aux expressions :

$$
\begin{aligned}
& i_{\mathrm{e}}(t)=\frac{E}{r+r_{\mathrm{e}}} \cdot\left[1-\left(\frac{\tau_{\mathrm{a}}-\tau_{1}}{\tau_{2}-\tau_{1}}\right) \cdot \mathrm{e}^{\left(-\frac{t}{\tau_{1}}\right)}+\left(\frac{\tau_{\mathrm{a}}-\tau_{2}}{\tau_{2}-\tau_{1}}\right) \cdot \mathrm{e}^{\left(-\frac{t}{\tau_{2}}\right)}\right] \\
& i_{\mathrm{a}}(t)=\frac{M_{\mathrm{ea}} \cdot E}{r_{\mathrm{a}} \cdot\left(r+r_{\mathrm{e}}\right)} \cdot \frac{1}{\tau_{2}-\tau_{1}} \cdot\left[\mathrm{e}^{\left(-\frac{t}{\tau_{1}}\right)}-\mathrm{e}^{\left(-\frac{t}{\tau_{2}}\right)}\right]
\end{aligned}
$$

avec :

avec :

$$
\begin{gathered}
\frac{1}{\tau_{1}}=\frac{r+r_{\mathrm{e}}}{2 \cdot \tau_{\mathrm{a}} \cdot L} \cdot\left[\tau_{\mathrm{p}}+\tau_{\mathrm{a}}+\sqrt{\left(\tau_{\mathrm{p}}+\tau_{\mathrm{a}}\right)^{2}-\frac{4 \cdot \tau_{\mathrm{a}} \cdot L}{r+r_{\mathrm{e}}}}\right] \\
\frac{1}{\tau_{2}}=\frac{r+r_{\mathrm{e}}}{2 \cdot \tau_{\mathrm{a}} \cdot L} \cdot\left[\tau_{\mathrm{p}}+\tau_{\mathrm{a}}-\sqrt{\left(\tau_{\mathrm{p}}+\tau_{\mathrm{a}}\right)^{2}-\frac{4 \cdot \tau_{\mathrm{a}} \cdot L}{r+r_{\mathrm{e}}}}\right] \\
\tau_{\mathrm{p}}=\frac{L+L_{\mathrm{e}}}{r+r_{\mathrm{e}}} \text { et } \tau_{\mathrm{a}}=\frac{L_{\mathrm{a}}}{r_{\mathrm{a}}}
\end{gathered}
$$

En utilisant les expressions (15) et (16), nous obtenons l'expression du flux :

$$
\phi(t)=\frac{E \cdot L_{\mathrm{e}}}{r+r_{\mathrm{e}}} \cdot\left[1+\left(\frac{\tau_{1}}{\tau_{2}-\tau_{1}}\right) \cdot \mathrm{e}^{\left(-\frac{t}{\tau_{1}}\right)}-\left(\frac{\tau_{2}}{\tau_{2}-\tau_{1}}\right) \cdot \mathrm{e}^{\left(-\frac{t}{\tau_{2}}\right)}\right]
$$


La f.e.m. de transformation induite dans une section d'induit est donnée par :

$$
e_{\mathrm{ts}}(t)=-n_{\mathrm{s}} \cdot \frac{\mathrm{d} \phi}{\mathrm{d} t}
$$

avec $n_{\mathrm{s}}$ : nombre de spires d'une section d'induit.

La dérivée de la relation (19) permet d'exprimer la f.e.m. de transformation selon :

$$
e_{\mathrm{ts}}(t)=\frac{E \cdot L_{\mathrm{e}} \cdot n_{\mathrm{s}}}{r+r_{\mathrm{e}}} \cdot \frac{1}{\tau_{2}-\tau_{1}} \cdot\left[\mathrm{e}^{\left(-\frac{t}{\tau_{1}}\right)}-\mathrm{e}^{\left(-\frac{t}{\tau_{2}}\right)}\right] .
$$

Toutes ces relations sont exploitées dans la simulation de l'amortisseur en régime dynamique.

L'expérimentation en régime dynamique nous donne la constante de temps électrique pour différentes valeurs de la résistance de l'amortisseur. Cette constante de temps est définie de la façon suivante : à l'instant $t=t_{0}$, un échelon de tension est appliqué aux bornes du circuit d'excitation. Cet échelon de tension provoque une variation de flux. On définit la constante de temps, comme étant le temps nécessaire pour que le flux atteigne $90 \%$ de sa valeur finale.

Le circuit d'excitation est alimenté par un générateur de signaux carrés suivi d'un amplificateur, ce signal d'excitation est noté $e(t)$.

Les signaux des tensions et courants sont obtenus par des acquisitions numériques via un oscilloscope interfacé IEEE.

Le flux est obtenu par intégration numérique de la f.e.m. de transformation. L'examen de la figure 5 montre que le modèle donne des résultats en bon accord avec l'expérience.

On retient comme paramètre typique, la constante de temps électrique.

\section{Recherche de l'amortisseur optimisé.}

La recherche de l'amortisseur optimisé est basée sur le modèle établi dans l'étude en régime stationnaire, du fonctionnement de la machine alimentée par un générateur de tension, en présence de l'amortisseur.

Dans cette étude, on a pu constater l'effet de la variation de la résistance de l'amortisseur ainsi que celle du nombre de spires le constituant sur les pertes et la f.e.m. de transformation.

Le dimensionnement de l'amortisseur s'établit en fonction de deux paramètres qui sont : $L_{\mathrm{a}}$ et $r_{\mathrm{a}}$.

L'inductance de l'amortisseur $L_{\mathrm{a}}$ est proportionnelle au carré du nombre de spires et la résistance au nombre de spires.

Ainsi, il existe un lien entre ces deux paramètres. Ce lien est la section totale de l'enroulement amortisseur.

Pour une section totale donnée de l'enroulement amortisseur, la f.e.m. de transformation dans une section en position de commutation, la constante de temps électrique, les pertes Joule du circuit d'excitation et les pertes Joule de l'amortisseur sont constantes quelle que soit la valeur de la résistance ou le nombre de spires de l'amortisseur.

Cette constatation est intéressante car elle permet de définir l'amortisseur le mieux adapté selon un parametre unique : la section totale de l'amortisseur.

La recherche de l'amortisseur le mieux adapté à notre machine se traduit par la figure 6 qui représente :

- les pertes Joule du circuit d'excitation;

- les pertes Joule de l'amortisseur ;

- la f.e.m. de transformation dans une section;

- la constante de temps électrique de la machine. 

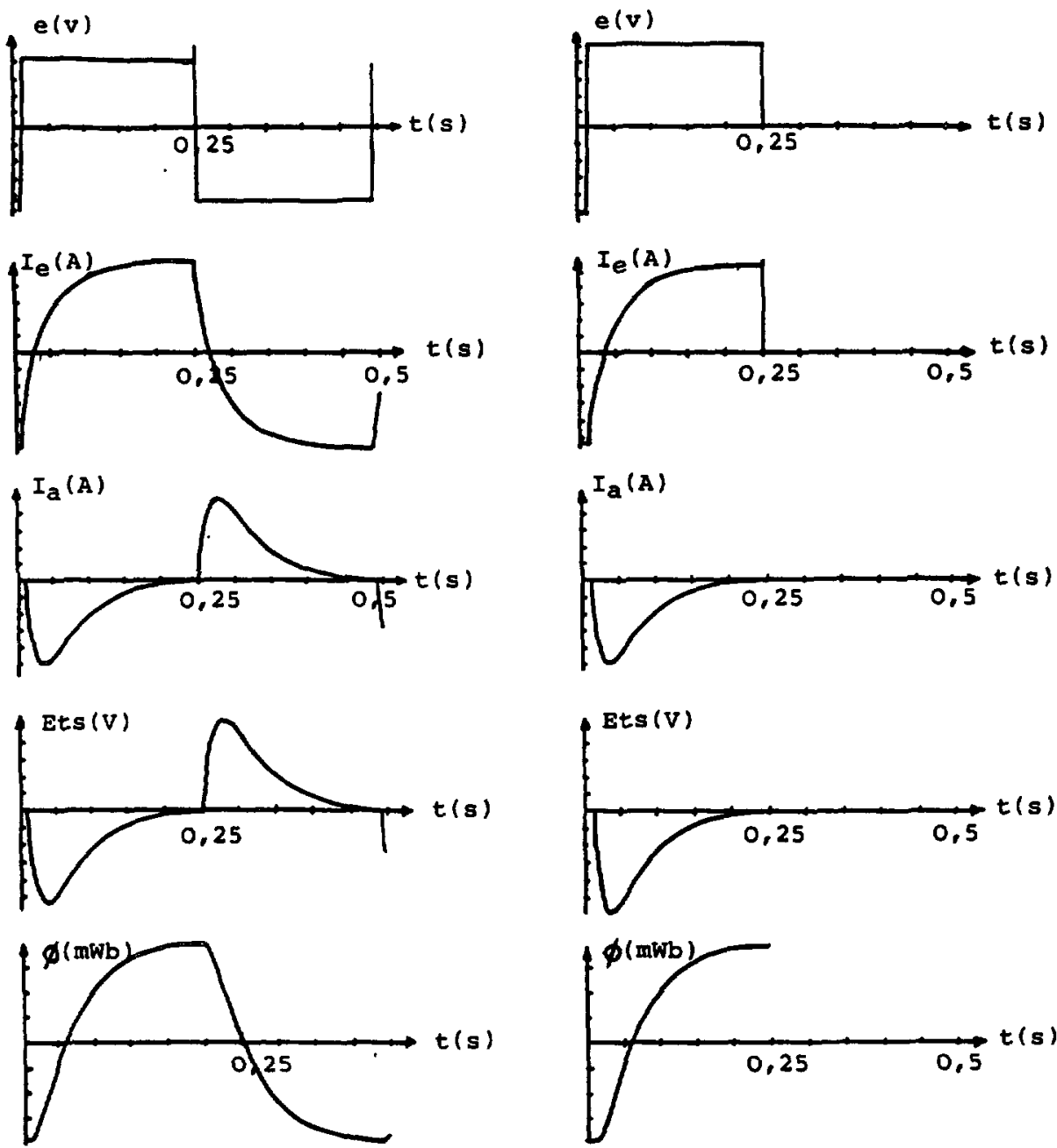

Expérimentation

rodele

Fig. 5. - Relevés théoriques et expérimentaux des grandeurs électriques et magnétiques. Circuit d'excitation : inducteur, induit et inductance de lissage. $r_{\mathrm{a}}=0,2 \Omega$.

[Theoretical and experimental curves of electrical and magnetic signals.]

Ces grandeurs sont calculées en fonction de la section totale de l'amortisseur, pour trois points de fonctionnement :

$$
U_{\mathrm{e}}=30 \mathrm{~V}\left\{\begin{array}{l}
U_{\mathrm{cc}}=10 \mathrm{~V} \\
U_{\mathrm{cc}}=20 \mathrm{~V} \\
U_{\mathrm{cc}}=30 \mathrm{~V}
\end{array}\right.
$$

La figure 6 montre bien que les pertes Joule ainsi que la f.e.m. de transformation tendent rapidement vers leurs asymptotes respectives avec la croissance de la section totale.

Cela signifie qu'au-delà d'une certaine limite, l'augmentation de la section n'a pour effet que d'accroître la constante de temps électrique et le volume global de l'amortisseur sans réaliser un gain important sur la f.e.m. de transformation. 


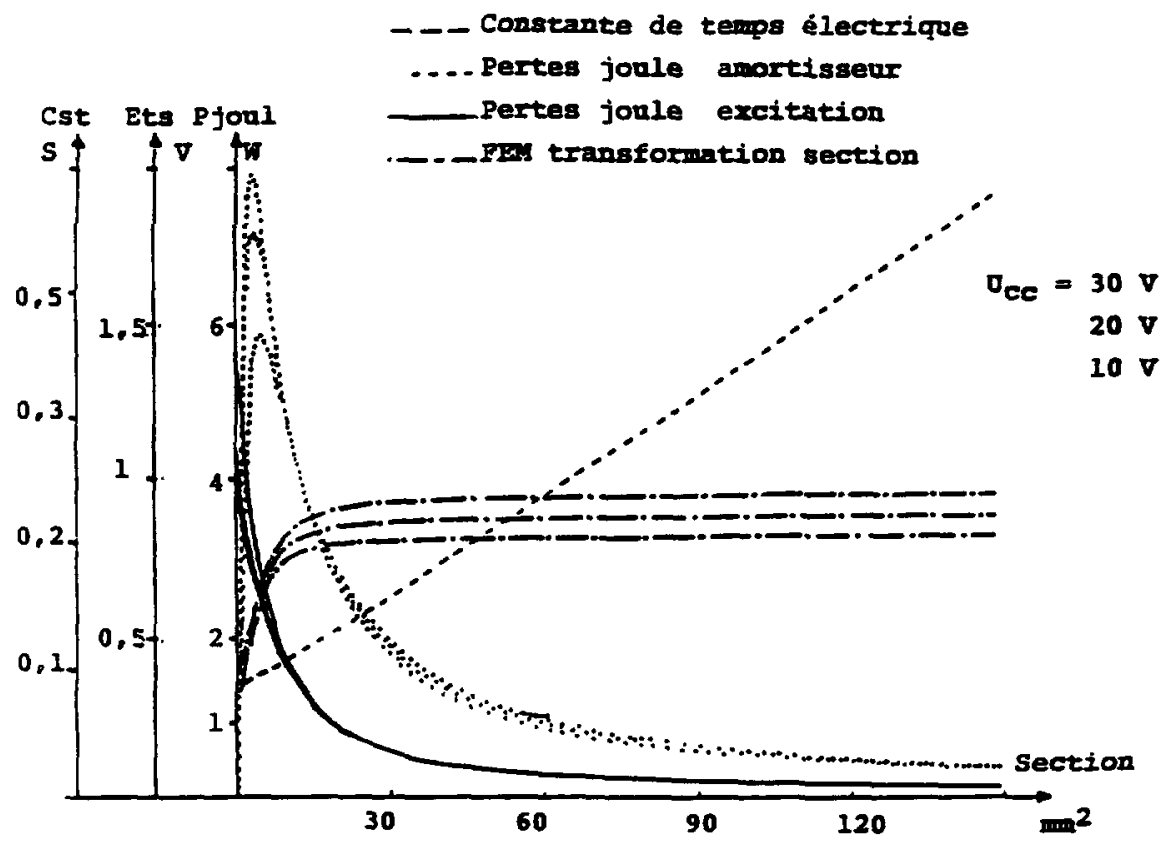

Fig. 6. - Variations des différents paramètres avec la section de totale de l'amortisseur. Inductance de lissage: $20 \mathrm{mH}, f=100 \mathrm{~Hz}, U_{\mathrm{e}}=30 \mathrm{~V}$.

[Parameter variations with the damper section.]

\section{Conclusion.}

La présence d'un enroulement amortisseur au sein de la machine a ses avantages et ses inconvénients.

- Le volume occupé par l'enroulement amortisseur et les pertes Joule supplémentaires sont les inconvénients majeurs. Avec une bonne optimisation ces deux facteurs peuvent être diminués. Dans le cas de notre machine, on constate que le volume de cuivre occupé par l'enroulement amortisseur est inférieur à $5 \%$ du volume de cuivre total des enroulements du stator et la somme estimée des pertes Joule du circuit d'excitation et de l'amortisseur est environ égale à $25 \mathrm{~W}$, ce qui représente moins de $1 \%$ de la puissance utile.

- La présence de l'amortisseur augmente la constante de temps électrique de la machine. Afin de ne pas affecter la réponse dynamique de la machine, il faut veiller à ne pas prendre une valeur trop proche de la valeur de la constante mécanique.

Du fait de la présence de l'amortisseur, le courant ondulé n'a pratiquement plus d'effet sur la commutation de la machine. Les étincelles au collecteur, les bruits d'origine magnétique et les pertes fer supplémentaires sont atténués. De ce fait, il est maintenant possible de diminuer l'inductance de lissage.

Ayant démontré l'efficacité de l'enroulement amortisseur, ce travail se prolonge par une étude en régime non sinusoïdal visant à optimiser l'inductance de lissage et à définir l'influence de l'amortisseur sur les couples pulsatoires.

Le rôle de l'enroulement amortisseur peut être joué par un stator entièrement ou partiellement massif : les courants induits dans le fer s'opposent aux variations du flux. L'optimisation peut se faire à l'aide de logiciels de calcul des champs magnétiques. 


\section{Bibliographie}

[1] Gabsi M. K., Lécrivain M., Rialland J. F., Commutation in DC machines field by static converters, Third International Conference on Electrical Machines and drives, I.E.E., London (November, 1987).

[2] GaBSI M. K., Contribution à l'étude de la commutation dans les machines à courant continu associées à des convertisseurs statiques de puissance, thèse de doctorat Paris 6 (1987).

[3] Budig G. K., Contribution to the calculation of the residual alternating current voltage in the commutating coil of the mixed current fed DC machine, Elektrie 34 (1980) 233-8.

[4] Gabsi M. K., Lécrivain M., Rialland J. F., Process to improve commutation in DC machines fed by static converters (ICEM, Pise, Italie, September, 1988).

[5] Peuro E., Caractérisation et optimisation d'un amortisseur destiné à améliorer la commutation d'une machine à courant continu alimentée par des convertisseurs statiques. Mémoire d'ingénieur CNAM (Novembre 1990).

[6] Dewolf F. T., Measurement of inductance of DC machines, IEEE Trans. Power Appar. Syst. PAS98 (1979) 1636-1644. 